\title{
Erratum to: Revisional Laparoscopic Gastric Pouch Resizing for Inadequate Weight Loss After Roux-en-Y Gastric Bypass
}

\author{
Ibtisam Al-Bader ${ }^{1}$ • Mousa Khoursheed ${ }^{1} \cdot$ Khalid Al Sharaf $^{2}$ • Ali Mouzannar ${ }^{2}$ • \\ Aqeel Ashraf $^{2}$ - Abe Fingerhut ${ }^{3,4}$
}

Published online: 5 May 2015

(C) Springer Science+Business Media New York 2015

Erratum to: OBES SURG

DOI 10.1007/s11695-015-1579-9

The name of author Ali Mouzannar was presented incorrectly in the article. The publisher apologizes for the error.

The online version of the original article can be found at http://dx.doi.org/ $10.1007 / \mathrm{s} 11695-015-1579-9$.

$\triangle$ Mousa Khoursheed

Khoursheed@hsc.edu.kw

1 Department of Surgery, Faculty of Medicine, Kuwait University, SafatPO Box 24923Jabriya 13110, Kuwait

2 Department of Surgery, Mubarak Al-Kabeer Hospital, Jabriya, Kuwait

3 First Department of Surgery, Hippocration Hospital, University of Athens Medical School, Athens, Greece

4 Section for Surgical Research, Clinical Division of General Surgery, Department of Surgery, Medical University of Graz, Graz, Austria 